\title{
MEGAMASERS AS PROBES OF GALAXY \\ MASS SPECTRUM EVOLUTION
}

\author{
V.K. KHERSONSKII ${ }^{1}$ AND N.V. VOSHCHINNIKOV ${ }^{2}$ \\ 1 Leningrad Department of Special Astrophysical Observatory, \\ 196140, Leningrad, Pulkovo, USSR \\ 2 Astronomical Observatory of Leningrad University, \\ 198904, Leningrad, Petrodvoretz, USSR
}

$\mathrm{OH}$ megamasers having very high luminosities in the spectral line can be effectively used for the probing of the evolutionary properties of the galaxies in the earliest cosmological epochs. The frequency shift of the emission line uniqually determines the redshift $z$, which tells about the epoch of emission. One of the important cosmological problems is the investigation of the galaxy mass spectrum in the expanding Universe. There is the empirical relation between the $\mathrm{OH}$ and farinfrared luminosities of galaxies. Therefore, if in the earliest cosmological epochs, there were galaxies with sufficient powerful infrared excesses and containing molecular material, they can be detected using the observations of their $\mathrm{OH}$ maser emission. The interacting and merging galaxies can be considered as the best candidates for such objects.

We determine the expected number of maser sources in the epoch at redshift $z$, using the model of galaxy mass spectrum (GMS) evolution in the expanding Universe. In this model, it is assumed that the GMS formation is connected with the collisions and mergers of galaxies and that these interactions are playing the dominant role in the initiation of the star formation process (Khersonskii and Voshchinnikov, 1991, to be published).

We estimate the number of expected $\mathrm{OH}$ maser sources in $1 \mathrm{Mpc}^{3}$ emitting in galaxies at the epoch of redshift $z, N_{O H}(z)$, for a given sensitivity of the radio observations. For the Arecibo radiotelescope, the ratio of this number to the total number density of galaxies at the epoch of redshift $z, S_{O H}(z)=N_{O H}(z) / n_{1}(t(z))$ increases with $z$ for values of the redshift smaller than 2 to 3 . Such a dependence of $S_{O H}(z)$ on $z$ is related to the general growth of the number of starbust galaxies. However, beyond some redshift $z_{1}, S_{O H}(z)$ decreases rapidly because of the absence of sufficiently massive galaxies in GMS. 